\title{
Beam test results for the SuperB-SVT thin striplet detector
}

L. Fabbri ${ }^{\text {c,d,* }}$, D. Comotti ${ }^{\mathrm{a}}$, M. Manghisoni ${ }^{\mathrm{a}, \mathrm{b}}, \mathrm{V} \cdot \operatorname{Re}^{\mathrm{a}, \mathrm{b}}, \mathrm{G}$. Traversi ${ }^{\mathrm{a}, \mathrm{b}}, \mathrm{A} \cdot$ Gabrielli $^{\mathrm{c}, \mathrm{d}}$, F. Giorgi ${ }^{\mathrm{d}}$, G. Pellegrini ${ }^{\mathrm{d}}$, C. Sbarra ${ }^{\mathrm{d}}$, N. Semprini-Cesari ${ }^{\mathrm{c}, \mathrm{d}}$, S. Valentinetti ${ }^{\mathrm{c}, \mathrm{d}}$, M. Villa ${ }^{\mathrm{c}, \mathrm{d}}$,

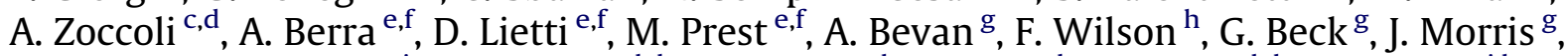
F. Ganaway ${ }^{g}$, R. Cenci ${ }^{i}$, L. Bombelli ${ }^{\mathrm{k}, 1}$, M. Citterio ${ }^{\mathrm{l}}$, S. Coelli ${ }^{1}$, C. Fiorini ${ }^{\mathrm{k}, 1}$, V. Liberali ${ }^{\mathrm{j}, \mathrm{l}}$, M. Monti ${ }^{1}$, B. Nasri ${ }^{k, 1}$, N. Neri ${ }^{1}$, F. Palombo ${ }^{\mathrm{j}, \mathrm{l}}$, A. Stabile $^{\mathrm{j}, \mathrm{l}}$, G. Balestri ${ }^{\mathrm{r}}$, G. Batignani ${ }^{\mathrm{p}, \mathrm{r}}$, A. Bernardelli ${ }^{r}$, S. Bettarini ${ }^{\mathrm{p}, \mathrm{r}}, \mathrm{F}$. Bosi ${ }^{\mathrm{r}}$, G. Casarosa ${ }^{\mathrm{p}, \mathrm{r}}$, M. Ceccanti $^{\mathrm{r}}$, F. Forti $^{\mathrm{p}, \mathrm{r}}$, M.A. Giorgi ${ }^{p, r}$, A. Lusiani ${ }^{q, r}$, P. Mammini ${ }^{r}$, F. Morsani ${ }^{r}$, B. Oberhof ${ }^{p, r}$, E. Paoloni ${ }^{p, r}$, A. Perez ${ }^{r}$, G. Petragnani ${ }^{r}$, A. Profeti $^{r}$, G. Rizzo ${ }^{\text {p,r }}$, A. Soldani ${ }^{r}$, J. Walsh ${ }^{r}$, L. Gaioni ${ }^{b}$,

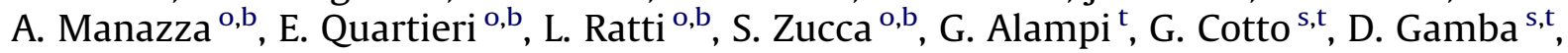
S. Zambito ${ }^{\mathrm{s}, \mathrm{t}}$, G.-F. Dalla Betta ${ }^{\mathrm{u}, \mathrm{n}}$, G. Fontana ${ }^{\mathrm{u}, \mathrm{n}}$, L. Pancheri ${ }^{\mathrm{u}, \mathrm{n}}$, M. Povoli ${ }^{\mathrm{u}, \mathrm{n}}$, G. Verzellesi ${ }^{\mathrm{n}, \mathrm{m}}$, M. Bomben $^{\mathrm{y}}{ }^{\text {, L. Bosisio }}{ }^{\mathrm{v}, \mathrm{w}}$, P. Cristaudo $^{\mathrm{w}}$, L. Lanceri ${ }^{\mathrm{v}, \mathrm{w}}$, B. Liberti ${ }^{\mathrm{w}}$, I. Rashevskaya ${ }^{w}$, C. Stella ${ }^{\mathrm{x}, \mathrm{w}}$, L. Vitale ${ }^{\mathrm{v}, \mathrm{w}}$

${ }^{a}$ Università degli Studi di Bergamo, Italy

${ }^{\mathrm{b}}$ Istituto Nazionale di Fisica Nucleare, Sezione di Pavia, Italy

${ }^{\mathrm{c}}$ Università degli Studi di Bologna, Italy

${ }^{\mathrm{d}}$ Istituto Nazionale di Fisica Nucleare, Sezione di Bologna, Italy

e Università dell'Insubria, Como, Italy

${ }^{\mathrm{f}}$ Istituto Nazionale di Fisica Nucleare, Sezione di Milano Bicocca, Italy

g School of Physics and Astronomy, Queen Mary University of London, London E1 4NS, UK

h STFC Rutherford Appleton Laboratory, Harwell Oxford, Didcot OX11 0QX, UK

${ }^{\mathrm{i}}$ University of Maryland, USA

${ }^{\mathrm{j}}$ Università degli Studi di Milano, Italy

${ }^{\mathrm{k}}$ Politecnico di Milano, Italy

${ }^{1}$ Istituto Nazionale di Fisica Nucleare, Sezione di Milano, Italy

${ }^{\mathrm{m}}$ Università degli Studi di Modena e Reggio Emilia, Italy

${ }^{\mathrm{n}}$ Istituto Nazionale di Fisica Nucleare, Sezione di Padova, Italy

- Università degli Studi di Pavia, Italy

P Università degli Studi di Pisa, Italy

q Scuola Normale Superiore, Pisa, Italy

${ }^{\mathrm{r}}$ Istituto Nazionale di Fisica Nucleare, Sezione di Pisa, Italy

${ }^{s}$ Università degli Studi di Torino, Italy

${ }^{\mathrm{t}}$ Istituto Nazionale di Fisica Nucleare, Sezione di Torino, Italy

u Università degli Studi di Trento, Italy

${ }^{v}$ Università degli Studi di Trieste, Italy

${ }^{\mathrm{w}}$ Istituto Nazionale di Fisica Nucleare, Sezione di Trieste, Italy

${ }^{x}$ Università degli Studi di Udine, Italy

${ }^{y}$ Laboratoire de Physique Nucléaire et de Hautes Energies, IN2P3/CNRS, Université Pierre et Marie Curie-Paris 6, Université Denis Diderot-Paris 7, F-75252 Paris, France

\section{A R T I C L E I N F O}

Available online 5 November 2012

Keywords:

Vertex detectors

Strip sensors

Striplets

Striplet detector

\begin{abstract}
A B S T R A C T
The baseline detector option for the first layer of the SuperB Silicon Vertex Tracker (SVT) is a high resistivity double-sided silicon device with short strips (striplets) at $45^{\circ}$ angle to the detector's edge. A prototype was tested with a $120 \mathrm{GeV} / c$ pion beam in September 2011 at the SPS-H6 test-beam line at CERN. In this paper studies on efficiency, resolution and cluster size are reported.
\end{abstract}

(c) 2012 Elsevier B.V. All rights reserved.

\footnotetext{
* Corresponding author at: Università degli Studi di Bologna, Italy. Tel.: + 390512091040.

E-mail address: laura.fabbri@bo.infn.it (L. Fabbri).
} 


\section{Introduction}

The SuperB factory [1] is expected to deliver luminosity in excess of $10^{36} \mathrm{~cm}^{-2} \mathrm{~s}^{-1}$ using a new beam steering technique that implies a reduced center-of-mass boost with respect to previous B-Factories (PEP-II [2] and KEKB [3]). This forces the SuperB detector to improve on typical vertex resolution. In order to achieve precise measurements near the interaction point, the SuperB Silicon Vertex Tracker (SVT) [4] will need a first layer very close to the beam pipe (Layer0). The requirements for the Layer0 will therefore be very stringent in terms of granularity, readout speed, material budget and radiation tolerance. Several options have been considered and in the most conservative one the Layer0 will be based on high resistivity sensors, with a thin silicon substrate and short double-sided strips. Furthermore, due to the device geometry, charged particles will have very high incident angles with the detector. A good spatial resolution at angles up to $70^{\circ}$ is an additional requirement.

\section{Beam test setup}

In September 2011 the SuperB collaboration tested low material budget silicon devices with the $120 \mathrm{GeV} / c$ pions SPS-H6 test-

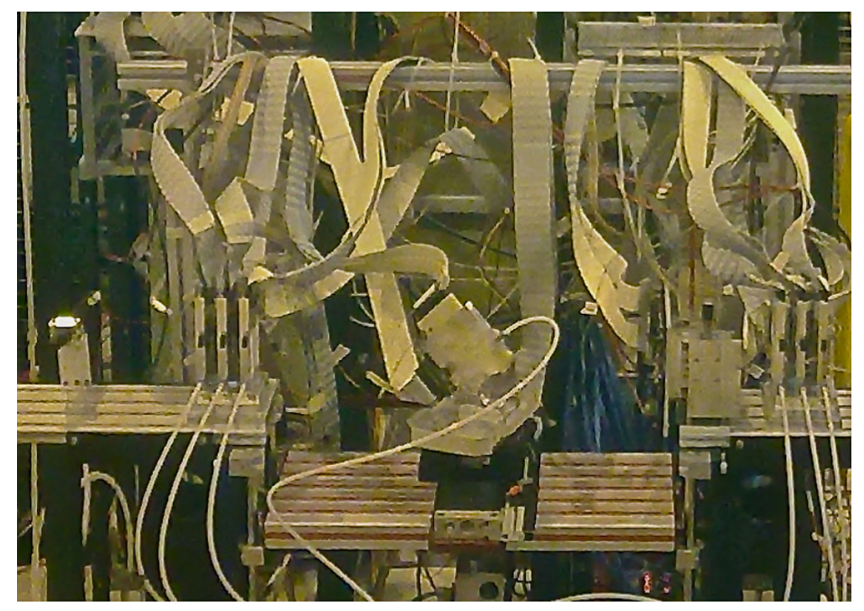

Fig. 1. Beam test setup. In the picture the DUT support rotated by $70^{\circ}$, the motorized table and the six telescope modules are shown. beam line at CERN. The Detectors Under Test (DUTs) were placed inside a high-resolution and fast-readout beam telescope.

The DUT is a device developed by the SLIM5 Collaboration [5]. It is a high resistivity double-sided silicon detector, $200 \mu \mathrm{m}$ thick, with $27 \times 12.9 \mathrm{~mm}^{2}$ active area, implemented with orthogonal short strips (striplets), $50 \mu \mathrm{m}$ pitch, tilted by $45^{\circ}$ to the detector's edge [6]. Up to two DUTs can be hosted and tested at the same time when they are situated orthogonally to the beam line. They can also be moved and rotated thanks to a motorized table equipped with a remote control, but in this case only one device can be tested (Fig. 1).

Two sets of thresholds are used. The first threshold (hit-no hit) is set to about $30 \%$ of the charge deposition of a minimum ionizing particle (MIP) for normal incidence in $200 \mu \mathrm{m}$ Silicon, corresponding to $20 \mathrm{ADC}$ counts, and it is compared to the lowest one set to about 20\% MIP, corresponding to 15 ADC counts. To check the performance at different angles of incidence several angular scans are carried out up to $70^{\circ}$ with respect to beam axis. For all the collected runs, single side efficiency, clusters size and resolution are studied.

The telescope is composed of six modules $300 \mu \mathrm{m}$ thick double-sided silicon strip detector with orthogonal strips. Three planes are placed before the DUTs and three after them. Each module consists of 384 channels per side, with an active area of $19 \times 19 \mathrm{~mm}^{2}$. The strips are characterized by $25 \mu \mathrm{m}$ pitch on p-side, with $50 \mu \mathrm{m}$ readout, $50 \mu \mathrm{m}$ pitch on n-side with a capacity of $4.3 \mathrm{pF}$. Telescope data are used to trigger good events and to find the impact point of tracks at the DUTs. By means of the telescope information the beam spot at DUT is reconstructed and horizontal and vertical widths of about 8 and $4 \mathrm{~mm}$ respectively are measured (Fig. 2).

Both the telescope strips and the striplets are read out by the FSSR2 Chip [7], completely data-driven. Each chip reads 128 strips with a digital output providing address, time stamp and three bit on pulse height. The readout clock operated at $\sim 20 \mathrm{MHz}$ allows a max data transmission rate of $240 \mathrm{Mbit} / \mathrm{s}$ over six lines.

\section{Event selection and alignment}

At least one hit on four telescope modules, two before and two after DUTs, is required to trigger an event. Moreover, one hit or more on each telescope module are required by track
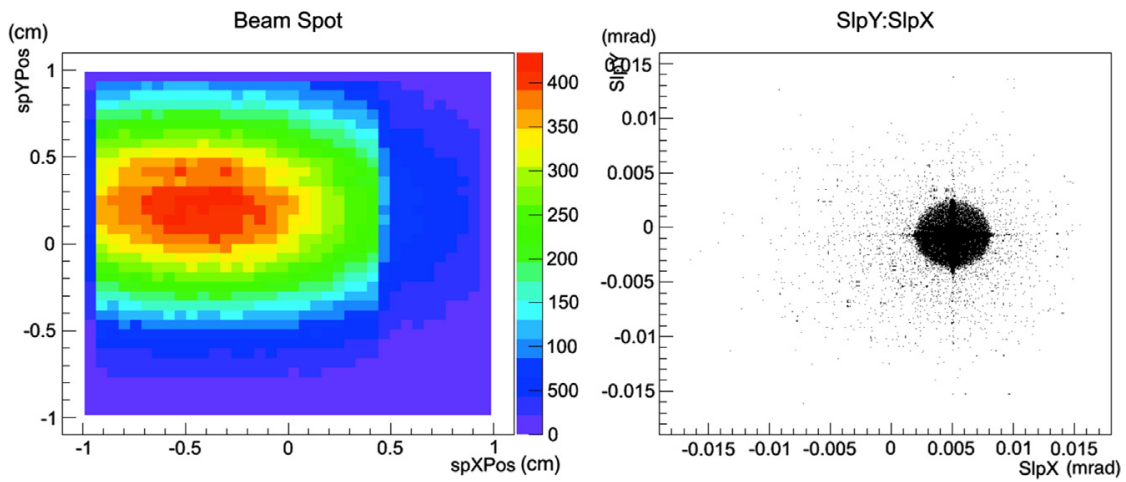

Fig. 2. Left: beam spot at DUT reconstructed by telescope. Right: beam slopes. 
reconstruction algorithm. The tracking method consist in building a road using the first and the last hits, in $z$ increasing order (where $z$ is the beam line axis), and requiring the four remaining hits to be less than $0.2 \mathrm{~mm}$ from the road. If this requirement is fulfilled least square method is used to extract track parameters. Only tracks with $\chi^{2}$ probability more than $10 \%$ are accepted.

Events with more than one track as well as events with tracks hitting inactive or hot strips and their closest channels are excluded from this analysis.

The first step of the analysis is the detectors alignment. It is an iterative process in which only events with only one track fulfilling $\chi^{2}$ requirement are taken into account. Residuals both for telescope and DUTs space points are computed in the strip directions ( $x$ and $y$ for telescope, $u$ and $v$ for DUTs, where $u$ and $v$ are orthogonal and in the striplets direction) and minimized via translations and rotations of the detectors. Typically, root mean square (RMS) values of DUT residual distributions at $0^{\circ}$ after detector alignment are of the order of $15(16) \mu \mathrm{m}$ at low (high) threshold. The minimum residuals are at $15^{\circ}$ (Fig. 4) and they increase drastically with the angle of incidence.

Due to the pions energy and the small thickness of the detectors the multiple scattering is negligible with respect to residuals and is not considered here.

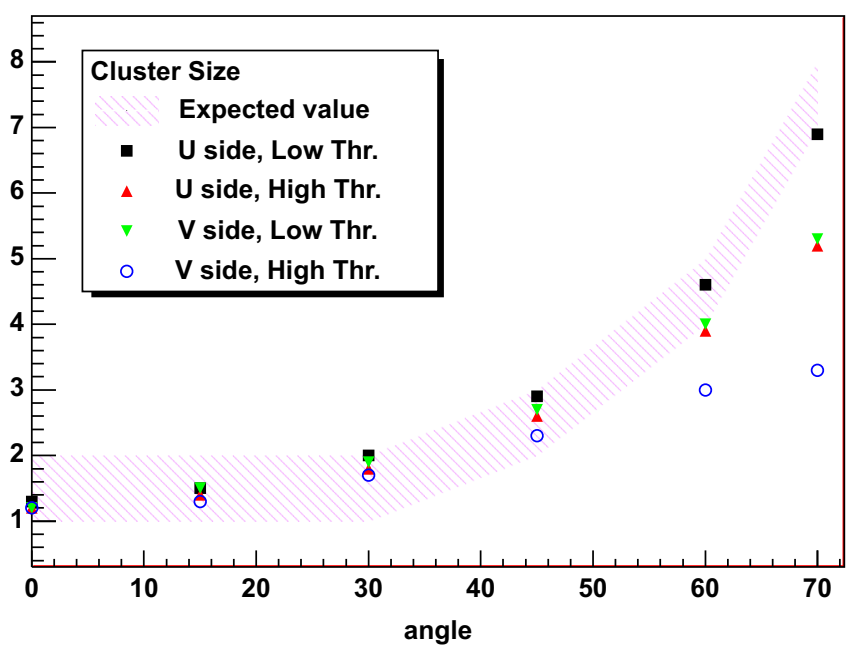

Fig. 3. Cluster size for $u$ and $v$ sides using low and high thresholds. The filled region represents the value estimated by pure geometrical considerations. Up to $30^{\circ}$ one or two channels results from particle impact point, over $30^{\circ}$ size increases for pure geometrical reason.

\section{Analysis and results}

The efficiency is defined as the percentage of events reconstructed by the DUT which are less than eight times the RMS to the detected track, corresponding to about $112 \mu \mathrm{m}$. For geometrical reason the distance projected on the DUT plane increases with the angle of incidence $\theta$. To take this effect into account the cut is scaled by the factor $\cos \theta$. The values obtained for the angular scan up to $70^{\circ}$ are better than $99.6 \%$ for lower threshold and $99.2 \%$ for the higher. Due to the huge amount of data the statistical error is negligible whereas the systematic uncertainty, estimated from different runs with the same settings, is of the order of $0.1 \%$.

A study on the size of clusters has also been performed. Ionization charges released by pions passing through the silicon device are usually collected by more than one striplet channel. The group of channels is a cluster, the weighted mean of strip positions and collected charges gives the hit coordinates. The number of striplets making a cluster (cluster size) increases with the angle of incidence, as expected by geometrical considerations. It is close to expectations at low threshold, as reported in Fig. 3, without significant increase due to noise. At high threshold the measured cluster size is far from the expected value. The lack of channels is due to the drop of charge collected by the single striplets because the charge is shared among more striplets and the probability for each of them to be above thresholds is lower. As a consequence, a percentage of the clusters are not completely reconstructed and their size results reduced. Increasing the threshold this effect is more evident.

The first estimate of resolution is taken from fit of residuals (Fig. 4). In Table 1 the RMSs of the residual histograms are reported for $u$ and $v$ sides, lower and higher thresholds and up to $70^{\circ}$.

Table 1

Resolution for $u$ and $v$ sides using low and high thresholds up to $70^{\circ}$ estimated as RMS of residual histogram.

\begin{tabular}{rllll}
\hline $\begin{array}{l}\theta \\
(\mathrm{deg})\end{array}$ & $\begin{array}{l}\sigma_{u} \text { Low thr. } \\
(\mu \mathrm{m})\end{array}$ & $\begin{array}{l}\sigma_{u} \text { High thr. } \\
(\mu \mathrm{m})\end{array}$ & $\begin{array}{l}\sigma_{v} \text { Low thr. } \\
(\mu \mathrm{m})\end{array}$ & $\begin{array}{l}\sigma_{v} \text { High thr. } \\
(\mu \mathrm{m})\end{array}$ \\
\hline 0 & 16.4 & 15.9 & 15.2 & 16.6 \\
15 & 12.4 & 13.9 & 13.4 & 15.6 \\
30 & 14.4 & 14.2 & 14.5 & 15.8 \\
45 & 18.3 & 17.6 & 21.0 & 22.8 \\
60 & 24.3 & 28.6 & 36.0 & 41.1 \\
70 & 36.8 & 52.3 & 56.3 & 62.0 \\
\hline
\end{tabular}

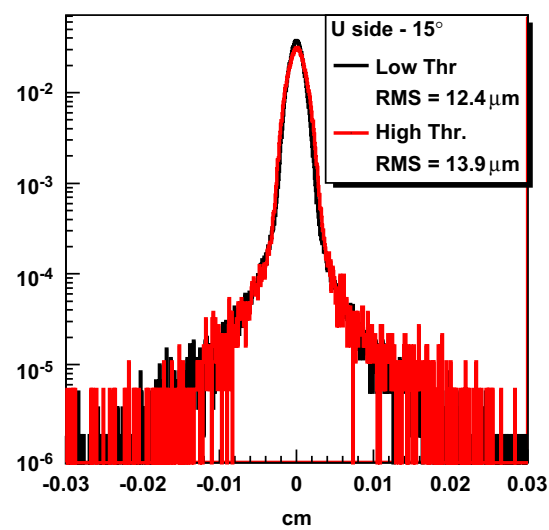

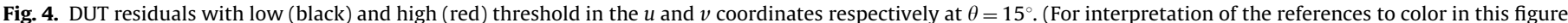
caption, the reader is referred to the web version of this article.) 


\section{Conclusions}

The low material budget prototype is tested with $120 \mathrm{GeV} / \mathrm{c}$ pions using different thresholds setting and for different incident track angles. Efficiency is studied as a function of both the threshold and the angle of incidence of the impinging pions. Both for lower and higher thresholds an efficiency better than $99 \%$ is measured up to $70^{\circ}$. Results from different runs are consistent within $0.1 \%$. These results are in good agreement with the previous ones obtained during 2009 beam test campaign performed at CERN-PS [6] where high threshold and $12 \mathrm{Gev} / \mathrm{c}$ pions were used. The cluster size is consistent with expectations considering reconstruction algorithm suffers from loss of channels under threshold for tracks with a large angle of incidence. The evaluation of resolution carried out using residuals RMS gives values better than $23 \mu \mathrm{m}$ up to $45^{\circ}$.

\section{References}

[1] The SuperB Conceptual Design Report, INFN/AE-07/02, SLAC-R-856, LAL 07-15, Available online at $\langle$ http://superb.infn.it/cdr $\rangle$.

[2] PEP-II Conceptual Design Report, SLAC-372, LBL-PUB-5303, CALT-68-1715, UCRL-ID-106426, UC-IIRPA-91-01, 1991; J. Seeman et al., SLAC-PUB- 12023, Contributed to European Particle Accelerator Conference (EPAC 06), Edinburgh, Scotland, 26-30 June 2006.

[3] S. Kurokawa, E. Kikutani, Nuclear Instruments and Methods in Physics Research A 499 (2003) 1. (and other papers of that volume).

[4] F. Forti, et al., Nuclear Instruments and Methods in Physics Research A (636) (2011) S168-S172.

[5] SLIM5 Collaboration-Silicon detectors with Low Interaction with Material $\langle$ http://www.pi.infn.it/slim5/〉.

[6] L. Vitale, et al., Nuclear Instruments and Methods in Physics Research A 617 (2010) 601;

M. Bomben, Nuclear Instruments and Methods in Physics Research A 623 (2010) 159;

S. Bettarini, et al., Nuclear Instruments and Methods in Physics Research A $623(2010) 942$.

[7] V. Re, M. Manghisoni, L. Ratti, J. Hoff, A. Mekkaoui, R. Yarema, IEEE Transactions on Nuclear Science NS-53 (August (4)) (2006) 2470. 